\title{
Nontuberculous mycobacteria isolated from specimens of pulmonary tuberculosis suspects, Northern Tunisia: 2002-2016
}

\author{
Reem Gharbi, Besma Mhenni, Saloua Ben Fraj and Helmi Mardassi* (i)
}

\begin{abstract}
Background: Reports on the worldwide ascending trend of pulmonary nontuberculous mycobacteria (NTM) isolation rates and their effective role in respiratory tract infections are compelling. However, as yet, there are no such data relating to Tunisia.

Methods: Here we carried out a retrospective review of mycobacterial cultures originating from Northern Tunisia, which have been processed in the laboratory of mycobacteria of the Institut Pasteur de Tunis, during the time period 2002-2016. All pulmonary NTM (PNTM) isolates available for culture were characterized phenotypically and their taxonomic status was further established based on polymorphisms in rpoB, 16S rRNA, hsp65, and sodA DNA gene sequences.

Results: Of the 10,466 specimens collected from HIV-negative Tunisian patients with presumptive clinical pulmonary TB, 60 (0.6\%) yielded PNTM isolates. An overall annual PNTM isolation prevalence of 0.2/100,000 was estimated. As far as could be ascertained, this isolation rate accounts amongst the lowest reported hitherto throughout the world. Among the 30 NTM isolates that were available for culture, 27 (90.0\%) have been identified to the species level. The most commonly encountered species was Mycobacterium kansasii (23.3\%) subtype 1. Strikingly, all M. kansasii cases were male patients originating from Bizerte, an industrialized region particularly known for iron industry. The remaining NTM species were M. fortuitum (16.6\%), M. novocastrense (16.6\%), M. chelonae (10.0\%), M. gordonae (6.6\%), M. gadium (6.6\%), M. peregrinum (3.3\%), M. porcinum (3.3\%), and M. flavescens (3.3\%). There were no bacteria of the M. avium complex, the most frequently isolated NTM globally, and the main driver of the rise of NTM-lung diseases.
\end{abstract}

Conclusions: This study uncovered an exceptional low prevalence of PNTM isolation among HIV-negative TB suspects in Northern Tunisia, suggesting a very low burden of NTM pulmonary disease. However, the frequent isolation of $M$. kansasii subtype 1, the most pathogenic subtype, particularly from the industrialized region of Bizerte, strongly suggests its effective involvement in a typical pulmonary disease.

Keywords: Nontuberculous mycobacteria, Pulmonary NTM, Pulmonary disease; molecular identification, Phylogeny, Tunisia

\footnotetext{
* Correspondence: helmi.merdassi@pasteur.rns.tn

Unit of Typing \& Genetics of Mycobacteria, Laboratory of Molecular

Microbiology, Vaccinology, and Biotechnology Development, Institut Pasteur

de Tunis, Université de Tunis El Manar, 13 Place Pasteur, BP74, 1002 Tunis,

Tunisia
}

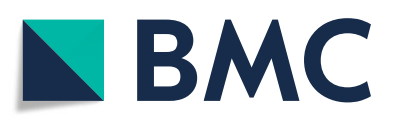

(0) The Author(s). 2019 Open Access This article is distributed under the terms of the Creative Commons Attribution 4.0 International License (http://creativecommons.org/licenses/by/4.0/), which permits unrestricted use, distribution, and reproduction in any medium, provided you give appropriate credit to the original author(s) and the source, provide a link to the Creative Commons license, and indicate if changes were made. The Creative Commons Public Domain Dedication waiver (http://creativecommons.org/publicdomain/zero/1.0/) applies to the data made available in this article, unless otherwise stated. 


\section{Background}

Mycobacteria encompass a large taxonomic group distributed in various aquatic and terrestrial environments. These are acid-fast bacilli (AFB) belonging to the genus Mycobacterium. Unlike obligate pathogenic species, Mycobacterium tuberculosis complex (MTBC) and $M$. leprae, the vast majority of mycobacteria are environmental organisms that usually act as opportunistic pathogens, causing localized (skin and soft tissues, lymph nodes, bones, lungs) or disseminated infections $[1,2]$. These opportunistic species are often collectively termed nontuberculous mycobacteria (NTM), but are also known as atypical mycobacteria, pathogenic environmental mycobacteria, or mycobacteria other than tuberculosis. NTM species are generally subdivided on the basis of growth into rapid and slow growing mycobacteria (RGM and SGM, respectively) [3]. Like MTBC members, pathogenicity in NTM is predominantly correlated with slow growth [4].

Given their ubiquitous presence in the environment, NTM can colonize humans, especially through the upper airways, and may cause a severe pulmonary disease in both immunocompromised and immunocompetent individuals $[5,6]$. Pulmonary infections due to NTM represent an emerging issue, particularly in Western countries where NTM prevalence may surpass TB [6]. There were significant differences in NTM species distribution within and between continents, which was suggested to influence the frequency and manifestations of pulmonary NTM (PNTM) disease in each geographical location [7].

In Tunisia, the national TB program (NTP) that was implemented in 1959 has significantly contributed to the overall decrease in TB incidence. Indeed, in the time period 1975 to 2002, TB incidence decreased from 48.6 down to 18.9 per 100,000 population. Yet, in the last decade, TB incidence has registered a steady increase, reaching 34.0 per 100,000 population in 2017 [8]. However, the magnitude of PNTM infection remains totally unknown.

While the geographical distribution of NTM isolation has been described worldwide [7], including subSaharan Africa [9], there are no data reported hitherto regarding Tunisia. Here, based on culture results of respiratory samples forwarded to the Institut Pasteur de Tunis during the time period 2002-2016, we estimated the prevalence of PNTM isolation among pulmonary TB suspects, originating mainly from Northern Tunisia, and determined the identity of the involved species.

\section{Methods}

\section{Pulmonary samples}

We carried out a retrospective review of mycobacterial cultures originating from Northern Tunisia (Tunis,
Bizerte, and Zaghouan), which have been processed in the laboratory of mycobacteria of the Institut Pasteur de Tunis, Tunisia. From January 2002 to November 2016, a total of 10,671 pulmonary samples obtained from TB suspects have been processed (Fig. 1). After excluding follow up, recurrent, and relapse cases, the culture results of the remaining 10,466 specimens (7699 sputum, 2093 bronchial washes, 674 pleural fluids) were taken into account.

Except for a few cases, all processed pulmonary samples were from Tunisian patients who permanently resided in Tunisia. The vast majority of patients from Tunis lived in urban districts, whereas the majority of patients from the regions of Bizerte, and particularly those from Zaghouan, resided in rural areas. Virtually, all TB suspects from the latter two regions were admitted into their respective unique regional hospital, and hence, their forwarded specimens represented a full capture of the prevailing TB situation. By contrast, patients from Tunis, came from various healthcare sites from both the public and private sectors.

\section{Mycobacterial isolation and phenotypic characterization} Briefly, pulmonary specimens (sputum, pleural fluid, bronchial wash) were processed by the $\mathrm{N}$-acetyl L cysteine-sodium hydroxide (NALC-NaOH) method, and inoculated into Löwenstein-Jensen (LJ) medium. Inoculated LJ slants were incubated at $37^{\circ} \mathrm{C}$ for eight weeks and examined every week.

Differentiation between MTBC and NTM colonies were initially performed by four standard biochemical tests: niacin, nitrate, heat-resistant catalase test (HRCT) and para- nitro benzoic acid (PNB). The NTMs were confirmed by PCR targeting the $\operatorname{rec} A$ intein, as previously described [10], and were further characterized phenotypically by morphological character and biochemical tests, i.e. colony morphology, growth rate, growth at various temperatures $\left(25^{\circ} \mathrm{C}, 37^{\circ} \mathrm{C}\right.$ and $\left.44{ }^{\circ} \mathrm{C}\right)$, pigment production in dark (schotochromogen), pigment production on exposure of light (photochromogen), absence of pigment production (non-chromogen), semi-quantitative Catalase test (SQCT), Thiophene2-carboxylic acid hydrazide (TCH) susceptibility test, Tween 80 hydrolysis, Aryl sulphatase test ( 3 days and 14 days), and $\beta$-Gal activities.

\section{Data analysis}

One isolate per patient was eligible for calculating PNTM isolation rate, which refers to the total number of PNTM divided by the total number of pulmonary specimens received in a year (annual rate) or during the study period (overall isolation rate). For patients with multiple PNTM isolations, we mostly took into consideration the very first isolate. PNTM isolation prevalence for the study period, or for a given year, 


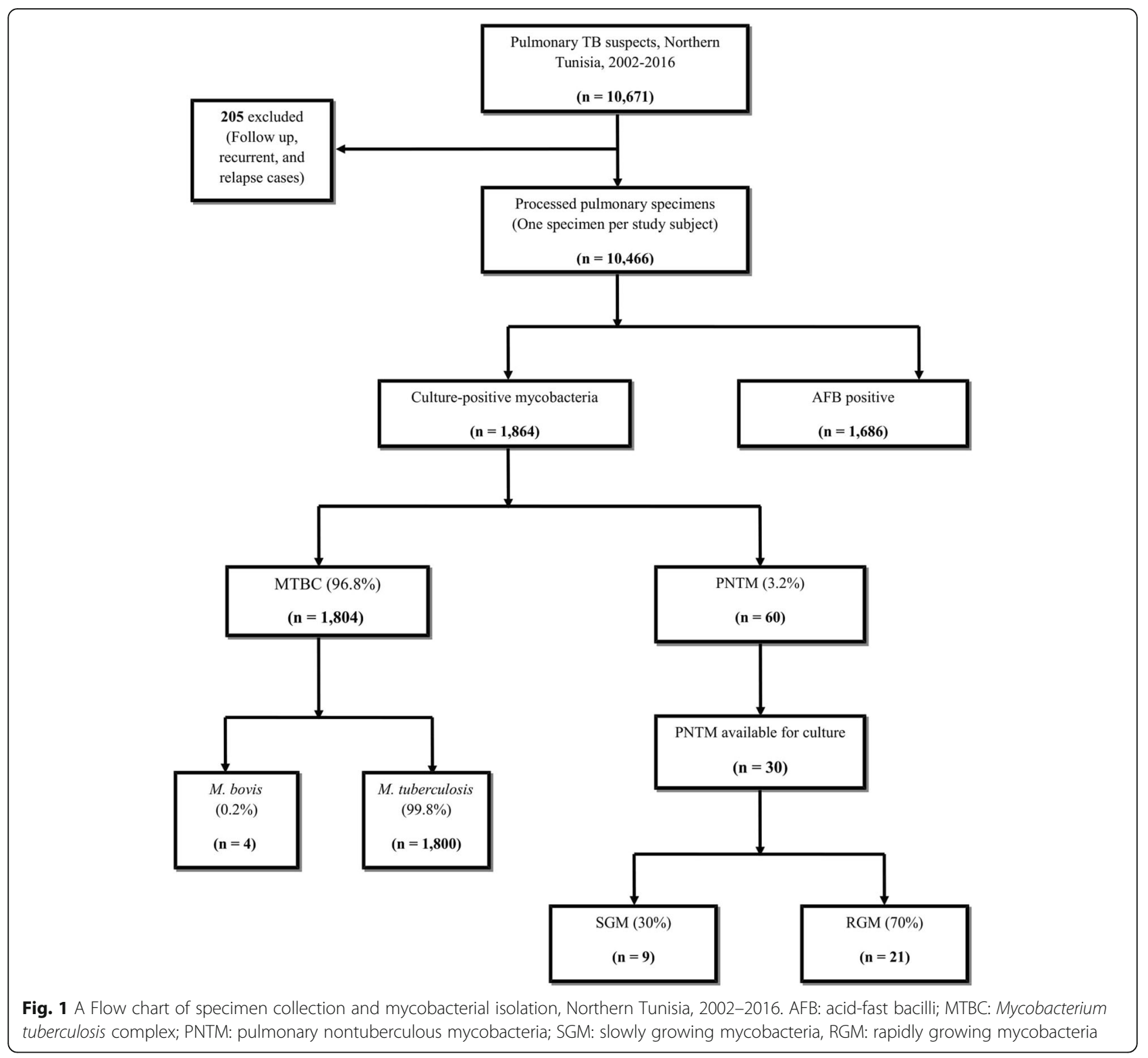

was calculated as the total number of PNTM positive cultures in a particular region divided by the population estimate and expressed per 100,000 population. In this study, we used the 2014 estimates of the Tunisian population, the most recent official estimates available: Tunis $(2,643,695$ inhabitants), Bizerte $(568,219$ inhabitants), and Zaghouan (176,945 inhabitants).

\section{PCR amplification and nucleotide sequencing}

From a few colonies cultivated on LJ slants, the DNA was extracted by thermolysis. For this purpose, the colonies are suspended in $100 \mu \mathrm{l}$ of 1\% EDTA / Triton Tris (TE / Triton) and inactivated at $80{ }^{\circ} \mathrm{C}$ for $30 \mathrm{~min}$.
PCR amplifications and sequencing of the rpoB, $16 \mathrm{~S}$ rRNA, hsp65, and sodA gene sequences were performed using the primer pairs listed in Additional file 1.

PCR reactions consisted of a15-min inactivation period followed by 35 cycles of $95^{\circ} \mathrm{C}$ for $30 \mathrm{~s}, 60{ }^{\circ} \mathrm{C}$ for $1 \mathrm{~min}\left(64{ }^{\circ} \mathrm{C}\right.$ for $1 \mathrm{~min}$ for $r p o B, 52^{\circ} \mathrm{C}$ for $30 \mathrm{~s}$ for $16 \mathrm{~S}$ rRNA) and $72^{\circ} \mathrm{C}$ for 2 min, with a final extension step at $72^{\circ} \mathrm{C}$ for $5-7 \mathrm{~min}$.

PCR amplifications were performed in a $50-\mu l$ PCR mixture containing $5 \mu \mathrm{l} \mathrm{10x}$ buffer (Qiagen, Courtaboeuf, France), $200 \mathrm{mM}$ each dNTP, $1.5 \mathrm{mM} \mathrm{MgCl}_{2}$, $1.25 \mathrm{U}$ HotStarTaq polymerase (Qiagen), $1 \mu \mathrm{l}$ each primer $(10 \mathrm{pM}), 33 \mu \mathrm{l}$ nuclease-free water and $5 \mu \mathrm{l}$ DNA template. Negative controls consisting of PCR mixture 
without DNA template were included in each PCR run. The PCR products were visualized by gel electrophoresis, treated with Shrimp Alkaline Phosphatase (SAP) and Exonuclease I (ExoI) (Sigma-Aldrich,USA), and sequenced in both directions using the BigDye Terminator sequencing kit (Applied Biosystems, Villebon-sur-Yvette, France) with an ABI PRISM 3100 automatic sequencer (ABI, USA). The sequences generated in the present study were deposited to the GeneBank. Their accession numbers are provided in Additional file 2.

Nucleotide sequences were processed using BioEdit (BioEdit software, version 7.0.5), and aligned with the GenBank database (NCBI) using the Basic Local Alignment Search Tool (BLAST).

\section{Species identification}

Species identification was primarily based on similarity rates with $r p o B$ gene sequence of reference strains (http:// www.bacterio.net/mycobacterium.html) [11]. Briefly, assignment of an isolate to a particular species was allowed if its rров sequence displayed at least 99.3\% similarity to type strain for slow growers and $98.3 \%$ for rapidly growing mycobacteria. Isolates showing similarity rates below these thresholds but $\geq 94 \%$ were identified to complex level. Isolates displaying less than 94\% similarity were referred to as rpoB unidentified Mycobacterium species (rUMS). With regard to $16 \mathrm{~S}$ rRNA gene sequencing, identification was based on $100 \%$ matches.

Subtyping of Mycobacterium kansasii was performed by PCR-restriction fragment length polymorphism (PCR-RFLP) analysis of the hsp65 gene as described previously [12].

\section{Phylogenetic analyses}

Phylogenetic analyses were carried out with MEGA (Molecular Evolutionary Genetics Analysis, version 7.0) [13]. Phylogenetic tree analyses were performed using the neighbor-joining method based on the Kimura twoparameter model with 1000 bootstrap replicates. Nocardia nova was used as the outgroup.

\section{Results}

Of the 10,466 specimens that were processed in the laboratory of Mycobacteria of the Institut Pasteur de Tunis for presumptive clinical pulmonary TB, 1864 mycobacteria-positive cultures were obtained, $96.8 \%$ of which belonged to the Mycobacterium tuberculosis complex (MTBC) (Fig. 1). PNTM isolation occurred in $60(3.2 \%)$ cases (54 isolates from sputum and 6 isolates from bronchial washes). All patients from whom PNTM isolates were obtained were HIV-negative, the majority of whom $(N=42,70.0 \%)$ were male. The distribution of isolated PNTMs according to the geographical region was as follows: 41 (68.0\%) from Tunis, 18 (30.0\%) from Bizerte, and 1 (2.0\%) from Zaghouan. The overall PNTM isolation rate was found to be $0.6 \%$ for the time period 2002-2016. PNTM isolation rates by region and/or per year are shown in Fig. 2a and Fig. 2b, respectively.

Since we ensured full coverage of the regions of Bizerte and Zaghouan during the time period 20022011, one can reliably estimate their respective mean annual prevalence of PNTM isolation, which were found to be $0.3 / 100,000$ and $0.1 / 100,000$, yielding an overall annual PNTM isolation prevalence of $0.2 / 100$, 000. It is worth noting that, during this time period, only one PNTM isolate was recovered from the 383 processed specimens from Zaghouan.

Initial classification of the cultured bacteria as NTM was based on their acid fastness and biochemical characteristics, and next confirmed by PCR targeting the rec $A$ intein (data not shown). Among the 30 cultured PNTM isolates, 9 (30.0\%) were slow growers. Phenotypic characteristics of the isolated PNTMs are shown in Table 1.

All PNTM isolates were subjected to rpoB, $16 \mathrm{~S}$ rRNA, hsp65 and sodA gene in an attempt to unambiguously assign a species to each of them. Based on BLAST analysis using rроB sequence data, following the previously adopted classification criteria [11], we could assign all but 2 isolates (90.0\% classification rate) to one of the following species/complex: M. kansasii, M. gordonae, M. fortuitum/M. fortuitum complex, $M$. Novocastrense, M. peregrinum, M. porcinum, M. flavescens, M. gadium, and M. chelonae (Table 2). In comparison, 16S rRNA sequencing allowed identifying 25 isolates to the species level (83.3\%) (Table 2).

Among the 9 SGM isolates, seven (77.7\%) were identified as M. kansasii, making up 23.3\% (7/30) of all PNTMs characterized in this study. All M. kansasii isolates were confirmed by $16 S r R N A$, hsp65, and $\operatorname{sod} A$ typing, since they displayed $100 \%$ similarity to the type strain, and all proved to be of subtype 1 (data not shown). The remaining two SGM isolates were identified as $M$. gordonae (100\% similarity to type strain for both $r p o B$ and $h s p 65$ gene sequences), which is consistent with their phenotypic characteristics.

The most commonly encountered species amongst the characterized RGM isolates $(N=21)$ were $M$. for tuitum $(N=5,23.8 \%), M$. novocastrense $(\mathrm{N}=5,23.8 \%)$, and $M$. chelonae $(N=3,14.3 \%)$, (Table 2). Indeed, these RGM isolates showed rpoB similarity rates $\geq 98.3 \%$ with their respective type strains. All $M$. fortuitum isolates were confirmed by both 16S rRNA and hsp65 sequencing (100\% similarity to type strain) (Table 2). One isolate, TNTM28, was considered as a 


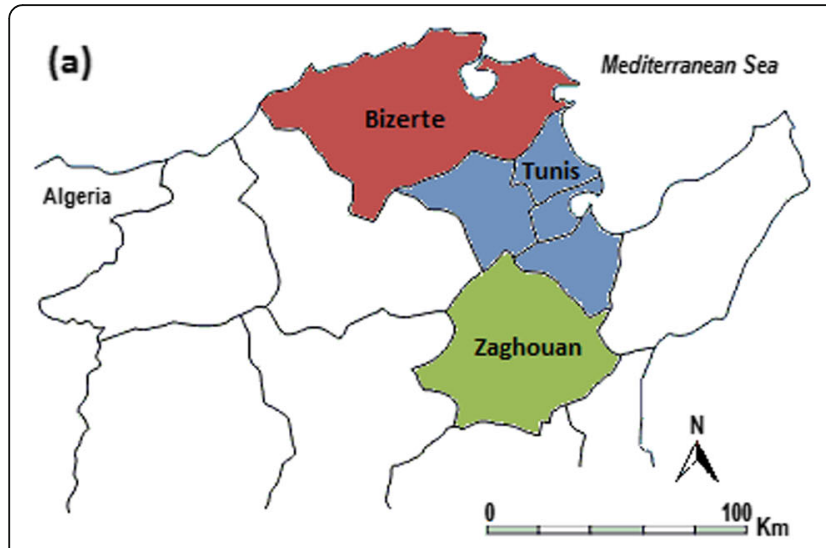

Isolation rate (\%)

(b)

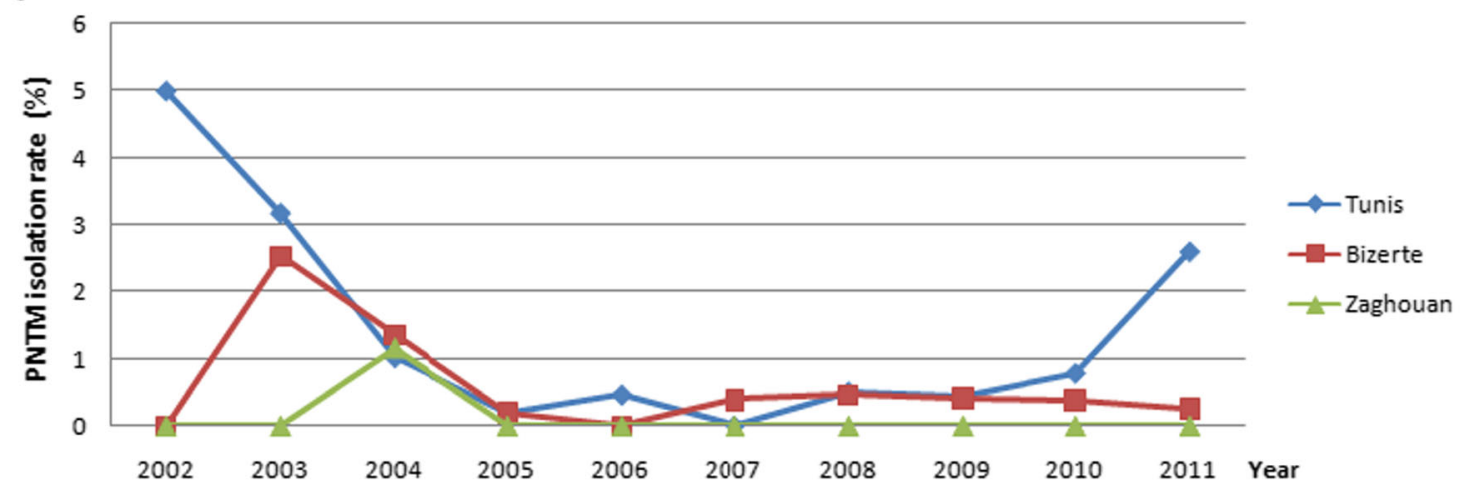

Fig. 2 (a) Map of Northern Tunisia showing PNTM isolation rates by region. The map was generated using Base map from https://d-maps.com; (b) Annual PNTM isolation rates (2002-2011) by region

member of the $M$. fortuitum complex based on $16 \mathrm{~S}$ rRNA $100 \%$ similarity with all $M$. fortuitum species. It remains to be seen whether it represents a new species within this complex. Of the $5 \mathrm{M}$. novocastrense isolates, only two could be confirmed by $16 \mathrm{~S}$ rRNA sequencing (100\% similarity to type strain). All M. chelonae isolates displayed $100 \%$ similarity to type strain at their hsp65 gene sequence. The remaining RGM isolates that were identified to the species level by rроB sequencing are $M$. gadium $(N=2,9.5 \%), M$. peregrinum $(N=1,4.8 \%), M$. porcinum $(\mathrm{N}=1,4.8 \%)$, and $M$. flavescens $(\mathrm{N}=1,4.8 \%)$.

Phylogenetic analyses using rpoB (Fig. 3), 16S rRNA (Additional file 3), and the rpoB-16S rRNA-hsp65-sodA concatenated sequence (Fig. 4) further confirmed the phylogenetic status of the 28 identified isolates.

Two RGM isolates could not be assigned a species or a complex, as they displayed less than $94 \%$ similarity to any known reference strain based on their rpoB sequence (Table 2). Furthermore, none of the two isolates showed $100 \%$ similarity to any known reference strain based on their $16 \mathrm{~S}$ rRNA, hsp65, or sodA gene sequences. The phylogenetic position of these two smooth chromogenic RGM isolates, TNTM29 and TNTM30, varied from one tree to another with low bootstrapping values. In all phylogenetic trees (Fig. 3, Additional file 3, and Fig. 4), TNTM29 and TNTM30 are the unique representative of their respective branch, generally occupying ancestral positions. Hence, the latter two isolates warrant further investigations as they may represent novel mycobacterial species.

\section{Discussion}

This study was primarily conducted to provide an insight into the extent of NTM involvement in respiratory tract infections in Northern Tunisia, and to uncover the identity of the isolated species. To our knowledge, this is the first study addressing this issue in North Africa. Our study covered a long time period of $\sim 15$ years and encompassed a large geographic area of Northern Tunisia, including the greatest Tunis (the capital and surrounding areas), as well as the regions of Bizerteand Zaghouan. Therefore, we believe it best captured the rates of NTM isolation among pulmonary $\mathrm{TB}$ suspects in the Tunisian population. 
Table 1 Epidemiologic and phenotypic features of NTM isolates recovered from Tunisian pulmonary TB suspects

\begin{tabular}{|c|c|c|c|c|c|c|c|c|c|c|c|c|c|c|}
\hline \multirow[t]{3}{*}{ Reference } & \multirow{3}{*}{$\begin{array}{l}\text { Year of } \\
\text { isolation }\end{array}$} & \multirow[t]{3}{*}{ Origin } & \multirow[t]{3}{*}{ Specimen } & \multirow[t]{3}{*}{ Sex } & \multirow{3}{*}{$\begin{array}{l}\text { Runyon } \\
\text { Classification }\end{array}$} & \multicolumn{9}{|c|}{ BIOCHEMICAL TESTS } \\
\hline & & & & & & \multirow[t]{2}{*}{ Niacin } & \multirow{2}{*}{$\begin{array}{l}\text { Nit- } \\
\text { Red }\end{array}$} & \multirow{2}{*}{$\begin{array}{l}\text { Urease } \\
3 \text { days }\end{array}$} & \multicolumn{2}{|c|}{ Catalase } & \multicolumn{2}{|c|}{ Arylsulfatase } & \multirow{2}{*}{$\begin{array}{l}\text { Tween } \\
80\end{array}$} & \multirow{2}{*}{$\begin{array}{l}\beta- \\
\text { Gal }\end{array}$} \\
\hline & & & & & & & & & $22^{\circ} \mathrm{C}$ & $68^{\circ} \mathrm{C}$ & 3 days & 14 days & & \\
\hline TNTM1 & 2002 & Bizerte & Sputum & M & P/SGM & - & + & + & + & + & - & + & + & - \\
\hline TNTM2 & 2009 & Bizerte & Sputum & M & P/SGM & - & + & + & + & + & - & $+/-$ & + & - \\
\hline TNTM3 & 2009 & Bizerte & Sputum & M & P/SGM & - & + & + & + & + & - & $+/-$ & + & - \\
\hline TNTM4 & 2010 & Bizerte & Sputum & M & P/SGM & - & + & + & + & + & - & $+/-$ & + & - \\
\hline TNTM5 & 2008 & Bizerte & Sputum & M & $\mathrm{P} / \mathrm{SGM}$ & - & + & + & + & + & - & + & + & - \\
\hline TNTM6 & 2002 & Bizerte & Sputum & M & P/SGM & - & + & + & + & + & - & + & + & - \\
\hline TNTM7 & 2007 & Bizerte & Sputum & M & P/SGM & - & + & + & + & + & - & + & + & - \\
\hline TNTM8 & 2000 & Tunis & Sputum & M & S/SGM & - & - & - & + & + & - & + & + & - \\
\hline TNTM9 & 2004 & Tunis & Sputum & M & S/SGM & - & - & - & + & + & - & + & + & - \\
\hline TNTM10 & 2003 & Tunis & Sputum & $\mathrm{F}$ & N/RGM & - & + & + & + & + & + & + & - & - \\
\hline TNTM11 & 2011 & Tunis & Sputum & M & N/RGM & - & + & + & + & + & + & + & - & - \\
\hline TNTM12 & 2015 & Tunis & Sputum & $\mathrm{F}$ & N/RGM & - & + & + & + & + & + & + & - & - \\
\hline TNTM13 & 2011 & Tunis & Sputum & $\mathrm{F}$ & $\mathrm{N} / \mathrm{RGM}$ & - & + & + & + & + & + & + & - & - \\
\hline TNTM14 & 2005 & Tunis & Sputum & M & N/RGM & - & + & + & + & + & + & + & - & - \\
\hline TNTM15 & 2004 & Tunis & Sputum & M & N/RGM & - & + & + & + & + & + & + & - & - \\
\hline TNTM16 & 2008 & Bizerte & Sputum & M & N/RGM & - & - & + & + & + & + & + & - & - \\
\hline TNTM17 & 2003 & Bizerte & Sputum & M & P/RGM & - & + & + & + & + & + & + & - & - \\
\hline TNTM18 & 2008 & Tunis & Sputum & M & P/RGM & - & + & - & + & + & + & + & - & - \\
\hline TNTM19 & 2008 & Tunis & Sputum & $\mathrm{F}$ & P/RGM & - & + & - & + & + & + & + & - & - \\
\hline TNTM20 & 2009 & Tunis & Sputum & M & P/RGM & - & + & + & + & + & + & + & - & - \\
\hline TNTM21 & 2004 & Tunis & Sputum & $\mathrm{F}$ & P/RGM & - & + & + & + & + & + & + & - & - \\
\hline TNTM22 & 2016 & Tunis & Sputum & M & S/RGM & - & + & + & + & + & + & + & + & - \\
\hline TNTM23 & 2003 & Bizerte & Sputum & M & S/RGM & - & + & + & + & + & - & - & - & - \\
\hline TNTM24 & 2003 & Tunis & Sputum & M & S/RGM & - & + & + & + & + & - & - & - & - \\
\hline TNTM25 & 2009 & Tunis & Sputum & M & N/RGM & - & - & + & + & + & + & + & - & - \\
\hline TNTM26 & 2010 & Tunis & Sputum & $\mathrm{F}$ & N/RGM & - & - & + & + & + & + & + & - & - \\
\hline TNTM27 & 2009 & Tunis & Sputum & M & $\mathrm{N} / \mathrm{RGM}$ & - & - & + & + & + & + & + & - & - \\
\hline TNTM28 & 2010 & Tunis & Sputum & M & N/RGM & - & + & + & + & + & + & + & - & - \\
\hline TNTM29 & 2004 & Tunis & Sputum & $\mathrm{F}$ & S/RGM & - & - & + & + & + & + & + & - & - \\
\hline TNTM30 & 2008 & Tunis & Sputum & M & $\mathrm{S} / \mathrm{RGM}$ & - & + & + & + & + & + & + & - & - \\
\hline
\end{tabular}

The overall PNTM isolation rate of $0.6 \%$ in Northern Tunisia was comparable to the rate of $1.0 \%$ previously reported among HIV-negative patients from Portugal [14]. However, these rates are low compared to the mean rate of $7.5 \%$ estimated for nine sub-Saharan African countries [9], or other regions where the prevalence of NTM isolation from pulmonary samples was $\sim 5$ to 16 times higher; being $2.6 \%$ in China (Shanghai) [15], 3.2\% in Iran [16], and 4.1 to $8.0 \%$ in Brazil $[17,18]$. Hence, NTM isolation from pulmonary samples in Northern Tunisia occurred very rarely with an overall annual prevalence of $0.17 / 100,000$. This rate is significantly low compared to Europe $(2.9-7.0 / 100,000)$ [19-23], as well as South and North America (5.3-22.2/ $100,000)[24-28]$. The low prevalence of NTM isolation in Northern Tunisia might stem from the fact that HIV infection is of very low prevalence $(\sim 0.1 \%)$ in this country [29]. Another explanation would be that for decades, the Tunisian population has been massively vaccinated with BCG, at birth and at entry to school, a practice that could have contributed to reduce NTM infection rates [30]. Furthermore, we believe that the use of the classical decontamination 
Table 2 Species assignments of Tunisian PNTM isolates according to \% similarity with rpoB, 16S rRNA, hsp65, and sodA gene sequences

\begin{tabular}{|c|c|c|c|c|}
\hline \multirow[t]{2}{*}{ Reference } & \multicolumn{4}{|c|}{ Species identification based on \% similarity } \\
\hline & $r p o B$ & 16S rRNA gene & hsp65 & sodA \\
\hline TNTM1 & M. kansasii (100\%) & M. kansasii (100\%) & M. kansasii (100\%) & M. kansasii (100\%) \\
\hline TNTM2 & M. kansasii (100\%) & M. kansasii (100\%) & M. kansasii (100\%) & M. kansasii (100\%) \\
\hline TNTM3 & M. kansasii (100\%) & M. kansasii (100\%) & M. kansasii (100\%) & M. kansasii (100\%) \\
\hline TNTM4 & M. kansasii (100\%) & M. kansasii (100\%) & M. kansasii (100\%) & M. kansasii (100\%) \\
\hline TNTM5 & M. kansasii (100\%) & M. kansasii (100\%) & M. kansasii (100\%) & M. kansasii (100\%) \\
\hline TNTM6 & M. kansasii (100\%) & M. kansasii (100\%) & M. kansasii (100\%) & M. kansasii (100\%) \\
\hline TNTM7 & M. kansasii (100\%) & M. kansasii (100\%) & M. kansasii (100\%) & M. kansasii (100\%) \\
\hline TNTM8 & M. gordonae (100\%) & M. gordonae (100\%) & M. gordonae (97.64\%) & M. gordonae (99.76\%) \\
\hline TNTM9 & M. gordonae (100\%) & M. gordonae (100\%) & M. gordonae (100\%) & M. gordonae (100\%) \\
\hline TNTM10 & M. fortuitum (100\%) & M. fortuitum (100\%) & M. fortuitum (100\%) & M.fortuitum (99.76\%) \\
\hline TNTM11 & M. fortuitum (100\%) & M. fortuitum (100\%) & M. fortuitum (100\%) & M. fortuitum (99.76\%) \\
\hline TNTM12 & M. fortuitum (100\%) & M. fortuitum (100\%) & M. fortuitum (100\%) & M. fortuitum (99.76\%) \\
\hline TNTM13 & M.fortuitum (100\%) & M. fortuitum (100\%) & M. fortuitum (100\%) & M. fortuitum $(99.76 \%$ \\
\hline TNTM14 & M.fortuitum (100\%) & M. fortuitum (100\%) & M. fortuitum 100\% & M. fortuitum (99.76\%) \\
\hline TNTM15 & M. peregrinum (100\%) & M. peregrinum (100\%) & M.peregrinum (100\%) & M. peregrinum (100\%) \\
\hline TNTM16 & M. porcinum (100\%) & M.porcinum (100\%) & M. porcinum (99.71\%) & M. porcinum (100\%) \\
\hline TNTM17 & M. novocastrense (99.99\%) & M. novocastrense (100\%) & M. novocastrense (100\%) & M. novocastrense (100\%) \\
\hline TNTM18 & M. novocastrense (100\%) & M. novocastrense (99.73\%) & M. novocastrense (100\%) & M. novocastrense (100\%) \\
\hline TNTM19 & M. novocastrense (100\%) & M. novocastrense (99.73\%) & M. novocastrense (100\%) & M. novocastrense (100\%) \\
\hline TNTM20 & M. novocastrense (99.85\%) & M. novocastrense (100\%) & M.novocastrense (100\%) & M. novocastrense (100\%) \\
\hline TNTM21 & M. novocastrense (99.85\%) & M. novocastrense (99.73\%) & M. novocastrense (100\%) & M. novocastrense (100\%) \\
\hline TNTM22 & M. flavescens (99.98\%) & M. flavescens (99.74\%) & M. flavescens (99.41\%) & M. flavescens (100\%) \\
\hline TNTM23 & M. gadium (100\%) & M. gadium (100\%) & M. gadium (100\%) & M. gadium (100\%) \\
\hline TNTM24 & M. gadium (98.45\%) & M. gadium (100\%) & M. gadium (98.82\%) & M. gadium (100\%) \\
\hline TNTM25 & M. chelonae (100\%) & M. chelonae (100\%) & M. chelonae 100\% & M. chelonae (100\%) \\
\hline TNTM26 & M. chelonae (100\%) & M. chelonae (100\%) & M. chelonae (100\%) & M. chelonae (100\%) \\
\hline TNTM27 & M. chelonae (100\%) & M. chelonae (100\%) & M. chelonae (100\%) & M. chelonae (100\%) \\
\hline TNTM28 & M. peregrinum (94.06\%) & M. fortuitum complex (100\%) & M. sp H12O-99,072 (96.76\%) & M. fortuitum (92.95\%) \\
\hline TNTM29 & rUMS (90.03\%) & M. sp GR2001-270 (100\%) & M. sp GR 2001-270 (97.64\%) & M. aurum (90.10\%) \\
\hline TNTM30 & rUMS (92.16\%) & M. sp G1368 (99.86\%) & M. sp G1368 (96\%) & M. abscessus (97.80\%) \\
\hline
\end{tabular}

protocol coupled to culture in Löwenstein-Jensen medium might not be optimal for NTM isolation [31, 32].

In this retrospective review we have not been able to extract reliable and complete clinical data in order to be able to estimate the real prevalence of NTM pulmonary disease. However, since it has been estimated that roughly $50 \%$, or even more less, of individuals whose respiratory cultures are positive for NTM fulfilled clinical criteria for NTM lung disease [5], one can rightfully argue that the overall prevalence of active respiratory infections due to NTM in Northern Tunisia is likely to be very low. Yet, this finding prompts a nationwide prospective study in order to determine the real prevalence of NTM respiratory disease in Tunisia.

Undoubtedly, the most common organism associated with pulmonary disease worldwide is by far the Mycobacterium avium complex (MAC), which includes at least 12 subspecies [33]. Pulmonary infection due to MAC was mainly associated with HIV infection [33]. The finding that no species belonging to MAC was isolated from our collection of respiratory samples is consistent with the very low prevalence $(\sim 0.1 \%)$ of HIV infection in Tunisia [29]. However, pulmonary disease due to MAC could also occur among HIV-negative patients. Hence, the rarity of 


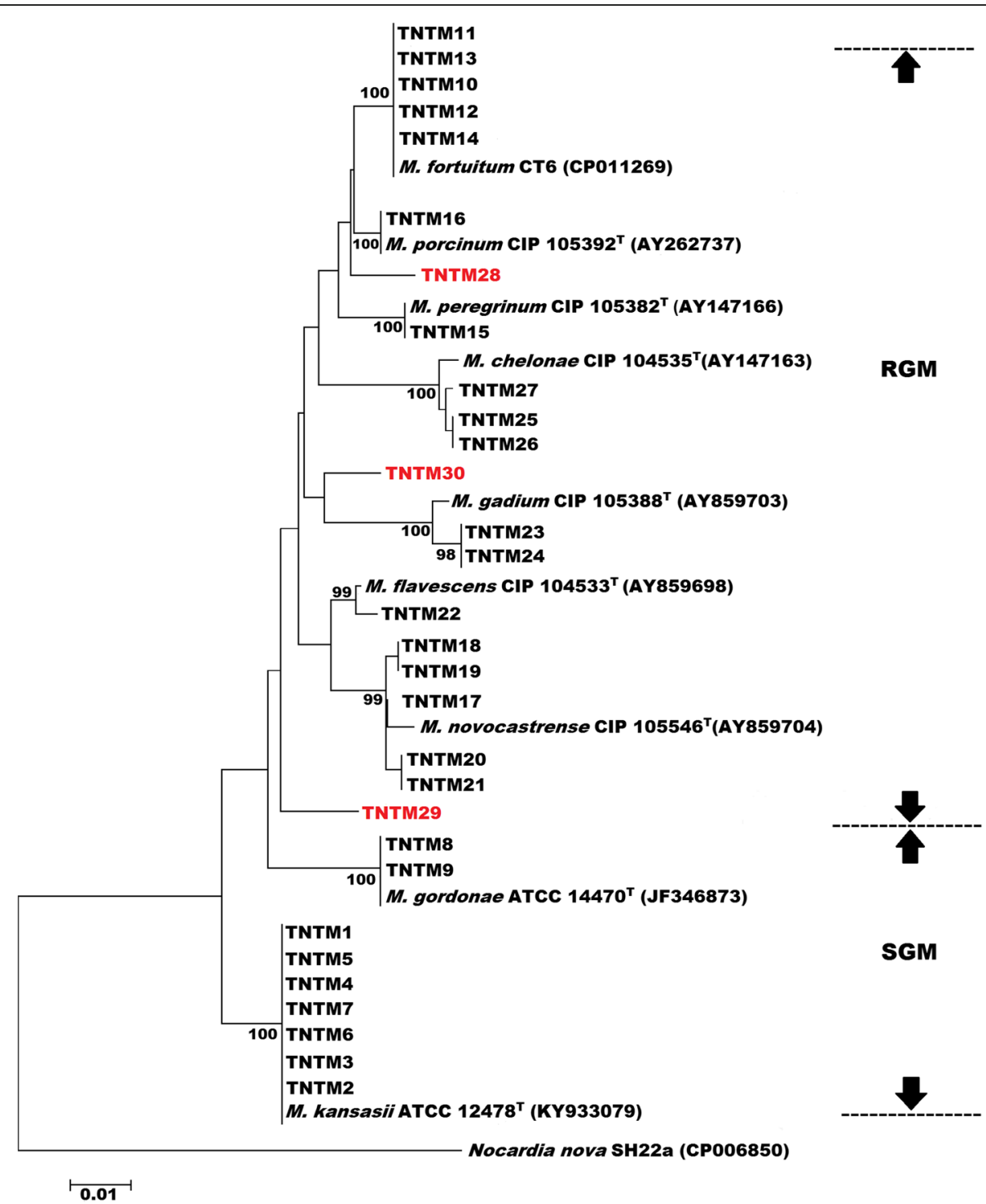

Fig. 3 Phylogenetic tree based on rpoB gene using NJ method with Kimura 2-parameter distance correction model. The significance of branches (when $>50$ ) is indicated by bootstrap values calculated on 1000 replicates. Bar, 1 substitution per 100 amino acid residues. PNTMs that could not be identified to the species level are highlighted in red

isolation of MAC species from Tunisian patients might also reflect a specific susceptibility pattern of the study population. In this respect, it is worth mentioning that important geographic variations in the species of isolated PNTM were observed throughout the world [7].

By contrast, the slow growing $M$. kansasii was the most frequently isolated NTM from respiratory specimens in northern Tunisia (23.3\%). M. kansasii is considered as one of the most pathogenic organism among all NTM species, and the presence of a single M. kansasii isolate in a sputum sample is generally interpreted as clinically significant [34]. This is particularly true in the United States and the United Kingdom where it was found that more than $70 \%$ of all patients with a respiratory isolate of $M$. kansasii had clinically relevant disease $[35,36]$. Aerosols generated from water systems of habitation and industries are likely to be the major source of this NTM, whose isolation has also been related to mining activities [37]. Strikingly, all M. kansasii cases reported in this study are men originating from $\mathrm{Bi}$ zerte, an industrialized region particularly known for iron industry. This observation warrants further investigation to understand the epidemiology of $M$. kansasii infection in this particular region and identify its potential reservoir. Furthermore, the finding that all $M$. kansasii clinical strains identified in Bizerte were of subtype 1, strongly suggests their involvement in a typical pulmonary disease [38]. 


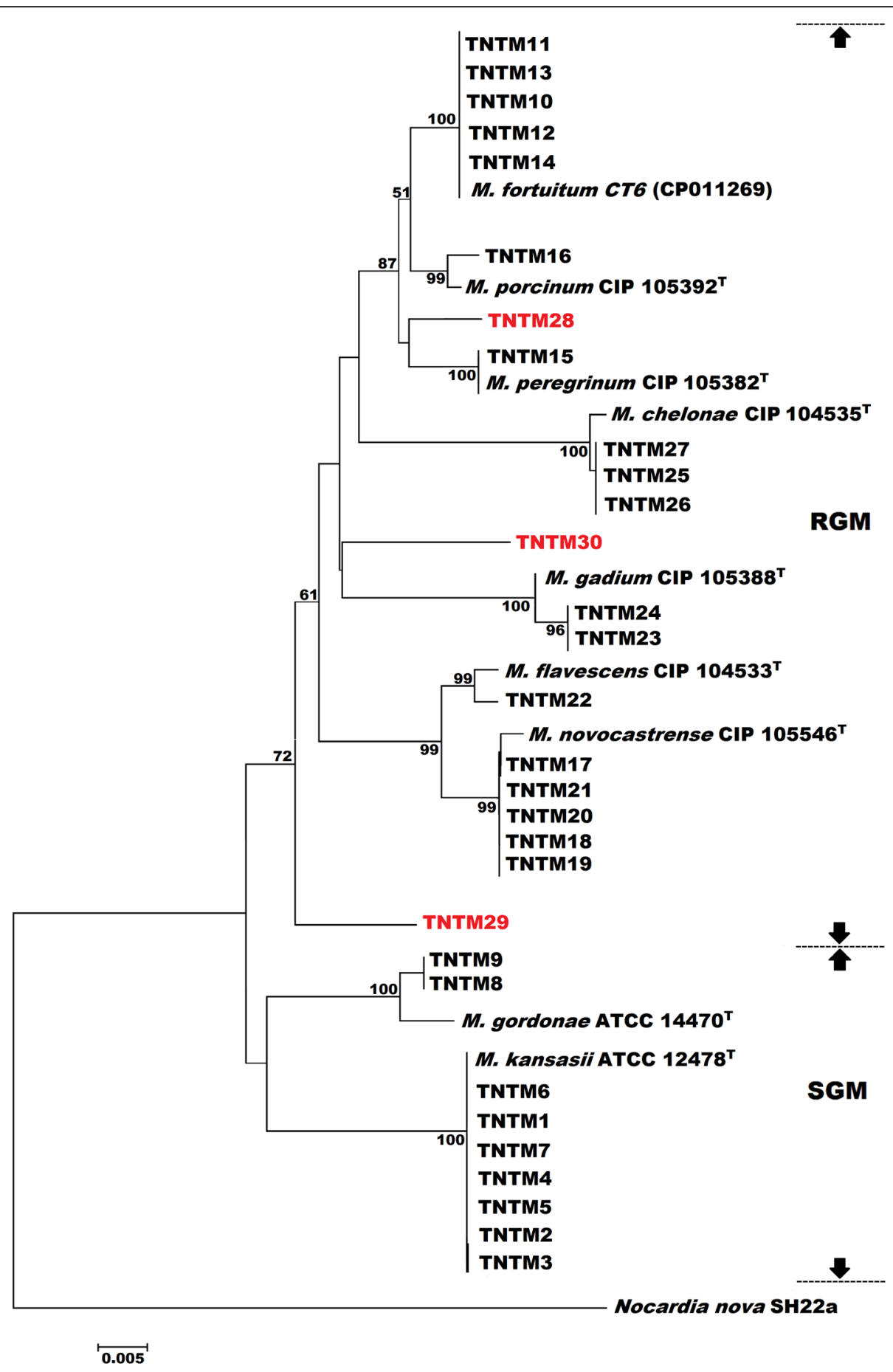

Fig. 4 Phylogenetic tree based on concatenated rpoB, 16S rRNA, hsp65 and sodA gene sequences using NJ method with Kimura 2-parameter distance model. The significance of branches (when $>50$ ) is indicated by bootstrap values calculated on 1000 replicates. Bar, 5 substitutions per 100 amino acid residues. PNTMs that could not be identified to the species level are highlighted in red

\section{Conclusion}

In summary, we provide the first insight into NTM pulmonary infection in Tunisia, reporting an exceptional low prevalence of PNTM isolation among HIV-negative $\mathrm{TB}$ suspects, thereby suggesting a very low burden of
NTM pulmonary disease. However, the frequent isolation of $M$. kansasii subtype 1 , the most pathogenic subtype, particularly from Bizerte, must alert the local health authorities therein in order to identify the source of infection. 


\section{Supplementary information}

Supplementary information accompanies this paper at https://doi.org/10. 1186/s12879-019-4441-1.

Additional file 1. List of primers used in this study.

Additional file 2. Genbank sequence accession numbers.

Additional file 3. Phylogenetic tree based on $16 \mathrm{~S}$ rRNA gene using NJ method with Kimura 2-parameter distance correction model. The significance of branches (when $>50$ ) is indicated by bootstrap values calculated on 1000 replicates. Bar, 5 substitutions per 1000 amino acid residues. PNTMs that could not be identified to the species level are highlighted in red.

\section{Abbreviations}

DNA: Deoxyribonucleic acid; MAC: Mycobacterium avium complex; MTBC: Mycobacterium tuberculosis complex; NTM: Non tuberculous mycobacteria; PCR: Polymerase chain reaction; PNTM: Pulmonary non tuberculous mycobacteria; RGM: Rapidly growing mycobacteria; SGM: Slowly growing mycobacteria

\section{Acknowledgements}

The authors are grateful to Mrs. Neila Khabouchi and Dr. Sinda Zarrouk for their valuable assistance with nucleotide sequencing.

\section{Authors' contributions}

All authors have made substantial contributions, RG and HM did the conception and design of the study, analysis and interpretation of data, drafting the manuscript. BM, SBF, and RG did the acquisition of data and laboratory work. RG and HM did the analysis of data and drafting of article. All authors approved the final version to be submitted.

\section{Authors' information}

Unit of Typing \& Genetics of Mycobacteria, Laboratory of Molecular Microbiology, Vaccinology, and Biotechnology Development. Institut Pasteur de Tunis, Université de Tunis El Manar. 13 Place Pasteur, BP74, 1002 Tunis, Tunisia.

\section{Funding}

This study received financial support from the Tunisian Ministry of Higher Education and Scientific Research (contract LR16IPT01) with no role in the design, data collection, analysis, or interpretation of the study and no role in writing this manuscript.

\section{Availability of data and materials}

The data and material included in this manuscript available from the corresponding author on reasonable request. The nucleotide data generated during the current study are available on GenBank nucleotide sequence database (https://www.ncbi.nlm.nih.gov/).

\section{Ethics approval and consent to participate}

No interventions were performed in this study. Only fully anonymized data were processed, ensuring the privacy for the individuals whose samples were analyzed. Therefore, no further ethical clearance was required. Furthermore, the samples used in this study have been collected as part of the routine diagnostic activity of the laboratory of Mycobacteria of the Institut Pasteur de Tunis, which is authorized by the Tunisian Ministry of Public Health, as part of the National TB program. Therefore, no administrative permissions were required to access the raw data.

\section{Consent for publication}

Not applicable.

\section{Competing interests}

The authors declare that they have no competing interest.
Received: 21 May 2019 Accepted: 4 September 2019

Published online: 18 September 2019

\section{References}

1. Honda JR, Virdi R, Chan ED. Global environmental nontuberculous mycobacteria and their contemporaneous man-made and natural niches. Front Microbiol 2018;9: 2029. PMID: 30214436

2. Wagner D, Young LS. Nontuberculous mycobacterial infections: a clinical review. Infection. 2004:32:257-270. PMID: 15624889.

3. Jarzembowski JA, Young MB. Nontuberculous mycobacterial infections. Arch Pathol Lab Med 2008;132:1333-1341. PMID: 18684037.

4. Leclerc MC, Thomas F, Guegan JF. Evidence for phylogenetic inheritance in pathogenicity of Mycobacterium. Antonie Van Leeuwenhoek. 2003;83:265-74.

5. Honda JR, Knight V, Chan ED. Pathogenesis and risk factors for nontuberculous mycobacterial lung disease. Clin Chest Med 2015;36:1-11. PMID: 25676515

6. Prevots DR, Marras TK. Epidemiology of human pulmonary infection with nontuberculous mycobacteria: a review. Clin Chest Med 2015;36:13-34. PMID: 25676516

7. Hoefsloot W, van Ingen J, Andrejak C, Angeby K, Bauriaud R, Bemer P, et al. The geographic diversity of nontuberculous mycobacteria isolated from pulmonary samples: an NTMNETcollaborative study. Eur Respir J. 2013;42: 1604-13.

8. World Health Organization. Global Tuberculosis Report. WHO. 2017:2017.

9. Okoi C, Anderson STB, Antonio M, Mulwa SN, Gehre F, Adetifa IMO. Nontuberculous mycobacteria isolated from pulmonary samples in sub-Saharan Africa - a systematic review and Meta analyses. Sci Rep 2017;7:12002. PMID: 28931915.

10. Saves I, Lewis LA, Westrelin F, Warren R, Daffé M, Masson JM. Specificities and functions of the recA and pps1 intein genes of Mycobacterium tuberculosis and application for diagnosis of tuberculosis. J Clin Microbiol 2002:40:943-950. PMID: 11880421.

11. de Zwaan R, van Ingen J, van Soolingen D. Utility of rpoB gene sequencing for identification of nontuberculous mycobacteria in the Netherlands. J Clin Microbiol 2014;52:2544-2551. PMID: 24808238.

12. Telenti A, Marchesi F, Balz M, Bally F, Böttger EC, Bodmer T. Rapid identification of mycobacteria to the species level by polymerase chain reaction and restriction enzyme analysis. J Clin Microbiol 1993;31:175-178. PMID: 8381805

13. Kumar S, Stecher G, Tamura K. MEGA7: molecular evolutionary genetics analysis version 7.0 for bigger datasets. Mol Biol Evol 2016;33:1870-1874. PMID: 27004904

14. Amorim A, Macedo R, Lopes A, Rodrigues I, Pereira E. Non-tuberculous mycobacteria in HIV-negative patients with pulmonary disease in Lisbon, Portugal Scand J Infect Dis 2010;42:626-628. PMID: 20632900.

15. Wu J, Zhang Y, Li J, Lin S, Wang L, Jiang Y, Pan Q, Shen X. Increase in nontuberculous mycobacteria isolated in Shanghai, China: results from a population-based study PLoS One 2014;9:e109736. PMID: 25330201.

16. Velayati AA, Farnia P, Mozafari M, Malekshahian D, Seif S, Rahideh S, et al. Molecular epidemiology of nontuberculous mycobacteria isolates from clinical and environmental sources of a metropolitan city. PLoS One 2014;9: e114428. PMID: 25485795

17. da Costa ARF, Lopes ML, de Sousa MS, Suffys PN, Sales LHM, Lima KVB. Pulmonary nontuberculosis mycobacterial infections in the state of Pará, an endemic region for tuberculosis in North of Brazil. Rijeka: In Pulmonary infection, INTECH Open Acess Publisher. p. 390-8.

18. Lima CA, Gomes HM, Oelemann MA, Ramos JP, Caldas PC, Campos CE, et al Nontuberculous mycobacteria in respiratory samples from patients with pulmonary tuberculosis in the state of Rondônia, Brazil Mem Inst Oswaldo Cruz 2013;108:457-462. PMID: 23827995.

19. Henry MT, Inamdar L, O'Riordain D, Schweiger M, Watson JP. Nontuberculous mycobacteria in non-HIV patients: epidemiology, treatment and response. Eur Respir J 2004;23:741-746. PMID: 15176690.

20. Gerogianni I, Papala M, Kostikas K, Petinaki E, Gourgoulianis KI. Epidemiology and clinical significance of mycobacterial respiratory infections in Central Greece. Int J Tuberc Lung Dis. 2008;12:807-812. PMID: 18544208.

21. van Ingen J, Bendien SA, de Lange WC, Hoefsloot W, Dekhuijzen PN, Boeree $\mathrm{MJ}$, et al. Clinical relevance of non-tuberculous mycobacteria isolated in the Nijmegen-Arnhem region, The Netherlands Thorax 2009;64:502-506. PMID: 19213773. 
22. Jankovic M, Samarzija M, Sabol I, Jakopovic M, Katalinic Jankovic V, Zmak L, et al. Geographical distribution and clinical relevance of non-tuberculous mycobacteria in Croatia. Int J Tuberc Lung Dis 2013;17:836-841. PMID: 23676172.

23. Andréjak C, Thomsen V $\varnothing$, Johansen IS, Riis A, Benfield TL, Duhaut $P$, et al. Nontuberculous pulmonary mycobacteriosis in Denmark: incidence and prognostic factors. Am J Respir Crit Care Med 2010;181:514-521. PMID: 20007929.

24. Marras TK, Chedore P, Ying AM, Jamieson F. Isolation prevalence of pulmonary non-tuberculous mycobacteria in Ontario, 1997 2003. Thorax. 2007:62:661-666. PMID: 17311842

25. Pedro Hda S, Pereira MI, Goloni Mdo R, Ueki SY, Chimara E. Nontuberculous mycobacteria isolated in São José do Rio Preto, Brazil between 1996 and 2005. J Bras Pneumol 2008;34:950-955. PMID: 19099102.

26. Cassidy PM, Hedberg K, Saulson A, McNelly E, Winthrop KL. Nontuberculous mycobacterial disease prevalence and risk factors: a changing epidemiology. Clin Infect Dis. 200915;49:e124-9. PMID: 19911942.

27. Al Houqani M, Jamieson F, Chedore P, Mehta M, May K, Marras TK. Isolation prevalence of pulmonary nontuberculous mycobacteria in Ontario in 2007. Can Respir J. 2011;18:19-24. https://doi.org/10.1155/2011/865831.

28. Marras TK, Mendelson D, Marchand-Austin A, May K, Jamieson FB. Pulmonary nontuberculous mycobacterial disease, Ontario, Canada, 19982010. Emerg Infect Dis 2013;19:1889-1891. doi: https://doi.org/10.3201/ eid1911.130737. PMID: 24210012

29. Gökengin D, Doroudi F, Tohme J, Collins B, Madani N. HIV/AIDS: trends in the Middle East and North Africa region. Int J Infect Dis 2016;44:66-73. PMID: 26948920

30. Zimmermann P, Finn A, Curtis N. Does BCG vaccination protect against nontuberculous mycobacterial infection? A systematic review and metaanalysis. J Infect Dis 2018;218:679-687. PMID: 29635431

31. Plongla R, Preece CL, Perry JD, Gilligan PH. Evaluation of RGM medium for isolation of nontuberculous mycobacteria from respiratory samples from patients with cystic fibrosis in the United States. J Clin Microbiol 2017:55: 1469-1477. PMID: 28228494.

32. Rotcheewaphan S, Odusanya OE, Henderson CM, Stephenson D, Olivier KN, Perry JD, et al. Performance of RGM medium for isolation of nontuberculous mycobacteria from respiratory specimens from non-cystic fibrosis patients. J Clin Microbiol 2019; 57(2): e01519-e01518. PMID: 30487305.

33. Field SK, Fisher D, Cowie RL. Mycobacterium avium complex pulmonary disease in patients without HIV infection. Chest. 2004;126:566-581. Review. PMID: 15302746

34. Griffith DE. Management of disease due to Mycobacterium kansasii. Clin Chest Med 2002;23: 613-621. PMID:12370997.

35. O'Brien RJ, Geiter LJ, Snider DE Jr. The epidemiology of nontuberculous mycobacterial diseases in the United States. Results from a national survey. Am Rev Respir Dis 1987;135:1007-1014. PMID: 3579001

36. Bloch KC, Zwerling L, Pletcher MJ, Hahn JA, Gerberding JL, Ostroff SM, et al. Incidence and clinical implications of isolation of Mycobacterium kansasii: results of a 5-year, population-based study. Ann Intern Med 1998;129: 698704. PMID: 9841601

37. van Halsema CL, Chihota VN, Gey van Pittius NC, Fielding KL, Lewis JJ, van Helden PD, et al. Clinical Relevance of Nontuberculous Mycobacteria Isolated from Sputum in a Gold Mining Workforce in South Africa: An Observational, Clinical Study. Biomed Res Int2015;2015:959107. PMID: 26180817.

38. Taillard C, Greub G, Weber R, Pfyffer GE, Bodmer T, Zimmerli S, et al. Clinical implications of Mycobacterium kansasii species heterogeneity: Swiss National Survey. J Clin Microbiol 2003;41:1240-1244. PMID: 12624057.

\section{Publisher's Note}

Springer Nature remains neutral with regard to jurisdictional claims in published maps and institutional affiliations.

\section{Ready to submit your research? Choose BMC and benefit from:}

- fast, convenient online submission

- thorough peer review by experienced researchers in your field

- rapid publication on acceptance

- support for research data, including large and complex data types

- gold Open Access which fosters wider collaboration and increased citations

- maximum visibility for your research: over $100 \mathrm{M}$ website views per year

At $\mathrm{BMC}$, research is always in progress.

Learn more biomedcentral.com/submissions 